\title{
Almost everywhere convergence of Marcinkiewicz means of Fourier series on the group of 2-adic integers
}

by

\section{Blahota and G. GÁt (Nyíregyháza)}

\begin{abstract}
We prove the almost everywhere convergence of the Marcinkiewicz means of integrable functions $\sigma_{n} f \rightarrow f$ for every $f \in L^{1}\left(I^{2}\right)$, where $I$ is the group of 2-adic integers.
\end{abstract}

We apply the standard notions of dyadic analysis as introduced by F. Schipp, P. Simon, W. R. Wade (see e.g. [7]) and others. Set $\mathbb{N}:=\{0,1, \ldots\}$, $\mathbb{P}:=\mathbb{N} \backslash\{0\}$, and $I:=[0,1)$. Denote by $\lambda(B)=|B|$ the Lebesgue measure of $B \subset I$, by $L^{p}(I)$ the usual Lebesgue spaces and $\|\cdot\|_{p}$ the corresponding norms $(1 \leq p \leq \infty)$. Let

$$
\mathcal{I}:=\left\{\left[\frac{p}{2^{n}}, \frac{p+1}{2^{n}}\right): p, n \in \mathbb{N}\right\}
$$

be the set of dyadic intervals, and for given $x \in I$ let $I_{n}(x) \in \mathcal{I}$ denote the interval of length $2^{-n}$ which contains $x(n \in \mathbb{N})$. Also set $I_{n}:=I_{n}(0)(n \in \mathbb{N})$. Let

$$
x=\sum_{n=0}^{\infty} x_{n} 2^{-(n+1)}
$$

be the dyadic expansion of $x \in I$, where $x_{n}=0$ or 1 , and if $x$ is a dyadic rational number $\left(x \in\left\{p / 2^{n}: p, n \in \mathbb{N}\right\}\right)$ we choose the expansion which terminates in 0 's.

The 2-adic (or arithmetic) sum of $a, b \in I$ is $a+b:=\sum_{n=0}^{\infty} r_{n} 2^{-(n+1)}$ where $q_{n}, r_{n} \in\{0,1\}(n \in \mathbb{N})$ are defined recursively as follows: $q_{-1}:=0$ and $a_{n}+b_{n}+q_{n-1}=2 q_{n}+r_{n}$ for $n \in \mathbb{N}$. (Since $q_{n}, r_{n}$ take on only the values 0,1 , these equations determine them uniquely.) The group $(I,+)$ is called the group of 2-adic integers. Set

$$
\varepsilon(t):=\exp (2 \pi \imath t) \quad(t \in \mathbb{R}),
$$

2000 Mathematics Subject Classification: 42C10, 42A24.

Key words and phrases: character system, group of 2-adic integers, Fourier series, Marcinkiewicz means, a.e. convergence. 
where $\imath=(-1)^{1 / 2}$. Set

$$
v_{2^{n}}(x):=\varepsilon\left(\frac{x_{n}}{2}+\cdots+\frac{x_{0}}{2^{n+1}}\right) \quad(x \in I, n \in \mathbb{N})
$$

and

$$
v_{n}:=\prod_{n=0}^{\infty} v_{2^{j}}^{n_{j}} \quad \text { for } n=\sum_{i=0}^{\infty} n_{i} 2^{i}\left(n_{i} \in\{0,1\}, i \in \mathbb{N}\right) .
$$

It is known [4] that the system $\left(v_{n}: n \in \mathbb{N}\right)$ is the character system of $(I,+)$. Denote by

$$
\widehat{f}(n):=\int_{I} f \bar{v}_{n} d \lambda, \quad D_{n}:=\sum_{k=0}^{n-1} v_{k}, \quad K_{n}:=\frac{1}{n} \sum_{k=0}^{n} D_{k}
$$

the Fourier coefficients, the Dirichlet kernels and the Fejér kernels, respectively. We will also use the notation

$$
K_{a, b}:=\sum_{k=a}^{a+b-1} D_{k} .
$$

It is known $[5,6,1]$ that for $n \in \mathbb{N}$ and $x \in I$,

$$
D_{2^{n}}(x)= \begin{cases}2^{n} & \text { if } x \in I_{n}, \\ 0 & \text { if } x \notin I_{n},\end{cases}
$$

and also that

$$
D_{n}(x)=v_{n}(x) \sum_{k=0}^{\infty} D_{2^{k}}(x) n_{k}(-1)^{x_{k}} .
$$

Next we introduce some notation for two-dimensional Fourier series on the group of 2-adic integers. The normalized Haar measure is just as in the one-dimensional case.

The two-dimensional Fourier coefficients, the rectangular partial sums of the Fourier series, the Marcinkiewicz means and the Marcinkiewicz kernels are defined as follows:

$$
\begin{gathered}
\widehat{f}\left(n^{1}, n^{2}\right):=\int_{I^{2}} f\left(x^{1}, x^{2}\right) \bar{v}_{n^{1}}\left(x^{1}\right) \bar{v}_{n^{2}}\left(x^{2}\right) d x \\
S_{n^{1}, n^{2}} f\left(x^{1}, x^{2}\right):=\sum_{k^{1}=0}^{n^{1}-1} \sum_{k^{2}=0}^{n^{2}-1} \widehat{f}\left(k^{1}, k^{2}\right) v_{k^{1}}\left(x^{1}\right) v_{k^{2}}\left(x^{2}\right), \\
\sigma_{n} f\left(x^{1}, x^{2}\right):=\frac{1}{n} \sum_{j=1}^{n} S_{j, j} f\left(x^{1}, x^{2}\right), \quad K_{n}\left(x^{1}, x^{2}\right)=\frac{1}{n} \sum_{j=1}^{n} D_{j}\left(x^{1}\right) D_{j}\left(x^{2}\right) .
\end{gathered}
$$


It is well known that for $y \in I^{2}$,

$$
\sigma_{n} f(y)=\int_{I^{2}} f(x) K_{n}(y-x) d x .
$$

The next notation will prove very useful:

$$
K_{a, b}\left(x^{1}, x^{2}\right):=\sum_{k=a}^{a+b-1} D_{k}\left(x^{1}\right) D_{k}\left(x^{2}\right) .
$$

Theorem 1. $\sigma_{n} f \rightarrow f$ for every $f \in L^{1}\left(I^{2}\right)$, where $I$ is the group of 2-adic integers.

This result for the trigonometric system was proved by Grünwald [2], and for some more general Nörlund means by Herriot [3]. See also the paper of Zhizhiashvili [8].

For $n, j \in \mathbb{N}$ let $n^{(j)}:=\sum_{i=j}^{\infty} n_{i} 2^{i}$, for $2^{B} \leq n<2^{B+1}$ let $|n|:=B$, and define $J_{\tau}:=I_{\tau} \backslash I_{\tau+1}$.

LEMMA 2. Let $t^{1} \leq t^{2}$. Then

$$
\sum_{t^{1}=0}^{m-1} \sum_{t^{2}=t^{1}}^{m-1} \int_{t^{1} \times J_{t^{2}}} \sup _{A \geq m} \sup _{|n|=A} \frac{1}{2^{A}} \sum_{s=t^{1}+1}^{A}\left|K_{n^{(s)}, 2^{s}}\left(x^{1}, x^{2}\right)\right| d x<c
$$

where $c$ is an absolute constant.

Proof. If $z \in J_{\tau}$ then

$$
K_{n^{(s)}, 2^{s}}(z)=\sum_{k=n^{(s)}}^{n^{(s)}+2^{s}-1} v_{k}(z)\left[\sum_{j=0}^{\tau-1} k_{j} 2^{j}+k_{\tau} 2^{\tau}(-1)\right] .
$$

Define $\widetilde{k}_{\tau}:=\sum_{j=0}^{\tau-1} k_{j} 2^{j}-k_{\tau} 2^{\tau}$. Then $\left|\widetilde{k}_{\tau}\right| \leq 2^{\tau}$.

In the two-dimensional case, if $x^{i} \in J_{t^{i}}(i=1,2)$ and $t^{1} \leq t^{2}$ then

$$
K_{n^{(s)}, 2^{s}}\left(x^{1}, x^{2}\right)=\sum_{k=n^{(s)}}^{n^{(s)}+2^{s}-1} v_{k^{\left(t^{1}+1\right)}}\left(x^{1}\right) v_{k^{\left(t^{2}+1\right)}}\left(x^{2}\right) \widetilde{k}_{t^{1}} \widetilde{k}_{t^{2}} .
$$

Assume $s>t^{1}$. Consider the following integral:

$$
\begin{aligned}
\int_{J_{\tau}} v_{k}(x) \bar{v}_{l}(x) d x & =\int_{I_{\tau} \backslash I_{\tau+1}} \prod_{j=0}^{\tau-1} v_{2^{j}}^{k_{j}}(x) \bar{v}_{2^{j}}^{l_{j}}(x)(-1)^{k^{\tau}+l^{\tau}} \prod_{j=\tau+1}^{\infty} v_{2^{j}}^{k_{j}}(x) \bar{v}_{2^{j}}^{l_{j}}(x) d x \\
& =(-1)^{k^{\tau}+l^{\tau}} \int_{I_{\tau+1}\left(l_{\tau}\right)} v_{k^{\tau+1}}(x) \bar{v}_{l^{\tau+1}}(x) d x \\
& = \begin{cases}(-1)^{k_{\tau}+l_{\tau} / 2^{\tau+1}} & \text { if } k^{(\tau+1)}=l^{(\tau+1)}, \\
0 & \text { otherwise. }\end{cases}
\end{aligned}
$$


From the Cauchy-Buniakovski inequality we have

$$
\begin{aligned}
\int_{J_{t^{1}} \times J_{t^{2}}}|f| & =\int_{I^{2}} 1_{J_{t^{1}} \times J_{t^{2}}} \cdot 1_{J_{t^{1}} \times J_{t^{2}}}|f| \leq\left\|1_{J_{t^{1}} \times J_{t^{2}}}\right\|_{2} \sqrt{\int_{I^{2}} 1_{J_{t^{1}} \times J_{t^{2}}}^{2}|f|} \\
& =\sqrt{\frac{1}{2^{t^{1}+t^{2}}} \int_{J_{t^{1}} \times J_{t^{2}}}|f|^{2} .}
\end{aligned}
$$

On the other hand, $\sup _{n}\left|K_{n^{(s)}, 2^{s}}\left(x^{1}, x^{2}\right)\right|$ depends only on $n_{s}, n_{s+1}, \ldots, n_{A}$ (for $|n|=A$ fixed).

Applying these facts we get

$$
\begin{aligned}
& \int_{J_{t^{1}} \times J_{t^{2}}} \sup _{n}\left|K_{n^{(s)}, 2^{s}}\left(x^{1}, x^{2}\right)\right| d x \leq \sqrt{\frac{1}{2^{t^{1}+t^{2}}} \int_{J^{1} \times J_{t^{2}}} \sup _{n}\left|K_{n^{(s)}, 2^{s}}\left(x^{1}, x^{2}\right)\right|^{2} d x} \\
& \leq \sqrt{\frac{1}{2^{t^{1}+t^{2}}} \int_{J_{t^{1}} \times J_{t^{2}}} \sum_{n_{s}, \ldots, n_{A} \in\{0,1\}}\left|K_{n^{(s)}, 2^{s}}\left(x^{1}, x^{2}\right)\right|^{2} d x} \\
& =\left(\frac{1}{2^{t^{1}+t^{2}}} \sum_{n_{s}, \ldots, n_{A} \in\{0,1\}} \sum_{k=n^{(s)}}^{n^{(s)} \sum_{l=2^{s}(s)}-1 n^{(s)}+2^{s}-1} \int_{J_{t^{1} \times J_{t^{2}}} v_{k^{\left(t^{1}+1\right)}}\left(x^{1}\right) \bar{v}_{l^{\left(t^{1}+1\right)}}\left(x^{1}\right)} \cdot v_{k^{\left(t^{2}+1\right)}}\left(x^{2}\right) \bar{v}_{l^{\left(t^{2}+1\right)}}\left(x^{2}\right) \widetilde{k}_{t^{1}} \widetilde{l}_{t^{1}} \widetilde{k}_{t^{2}} \widetilde{l}_{t^{2}} d x\right)^{1 / 2}
\end{aligned}
$$

These integrals are either zeros or can by estimated in absolute value by

$$
\frac{1}{2^{t^{1}+t^{2}}}\left|\widetilde{k}_{t^{1}} \widetilde{l}_{t^{1}} \widetilde{k}_{t^{2}} \widetilde{l}_{t^{2}}\right| \leq 2^{t^{1}+t^{2}} \text {. }
$$

The latter happens if $k^{\left(t^{1}+1\right)}=l^{\left(t^{1}+1\right)}$ and $k^{\left(t^{2}+1\right)}=l^{\left(t^{2}+1\right)}$, that is, exactly in case $k^{\left(t^{1}+1\right)}=l^{\left(t^{1}+1\right)}$, because $t^{1} \leq t^{2}$.

Let us count the number of such $(k, l)$ pairs. Since $k \in\left[n^{(s)}, n^{(s)}+2^{s}\right)$ and $k^{\left(t^{1}+1\right)}=l^{\left(t^{1}+1\right)}$, for every $k$ (their number is $2^{s}$ ) the number of $l$ 's satisfying the condition above is $2^{t^{1}+1}$. This yields altogether $2^{s+t^{1}+1}$ pairs $(k, l)$.

So we get

$$
\begin{aligned}
& \int_{t_{t^{1}} \times J_{t^{2}}} \sup _{n}\left|K_{n^{(s)}, 2^{s}}\left(x^{1}, x^{2}\right)\right| d x \\
& \leq\left(\frac{1}{2^{t^{1}+t^{2}}} \sum_{n_{s}, \ldots, n_{A} \in\{0,1\}} 2^{s+t^{1}+1} 2^{t^{1}+t^{2}}\right)^{1 / 2}=\left(2^{s+t^{1}+1} 2^{A-s+1}\right)^{1 / 2} \leq c \sqrt{2^{A+t^{1}}} .
\end{aligned}
$$


Using this inequality it follows that

$$
\begin{aligned}
& \sum_{t^{1}=0}^{m-1} \sum_{t^{2}=t^{1}}^{m-1} \int_{t^{1} \times J_{t^{2}}} \sup _{A \geq m} \sup _{|n|=A} \frac{1}{2^{A}} \sum_{s=t^{1}+1}^{A}\left|K_{n^{(s)}, 2^{s}}\left(x^{1}, x^{2}\right)\right| d x \\
& \quad \leq c \sum_{t^{1}=0}^{m-1} \sum_{t^{2}=t^{1}}^{m-1} \sum_{A=m \vee t^{2}}^{\infty} \sum_{s=t^{1}+1}^{A} \sqrt{2^{t^{1}-A}} \leq c \sum_{t^{1}=0}^{m-1} \sum_{t^{2}=t^{1}}^{m-1} \sum_{A=m \vee t^{2}}^{\infty}\left(A-t^{1}\right) \sqrt{2^{t^{1}-A}} \\
& \quad \leq c \sum_{t^{1}=0}^{m-1} \sum_{t^{2}=t^{1}}^{m-1}\left(m \vee t^{2}-t^{1}\right) \sqrt{2^{t^{1}-m}} \leq c \sum_{t^{1}=0}^{m-1}\left(m-t^{1}\right)^{2} \sqrt{2^{t^{1}-m}} \leq c .
\end{aligned}
$$

Moreover,

$$
\begin{aligned}
\sum_{t^{1}=0}^{m-1} & \sum_{t^{2}=m+1}^{\infty} \int_{J_{t^{1}} \times J_{t^{2}}} \sup _{t^{2}>A \geq m} \sup _{|n|=A} \frac{1}{2^{A}} \sum_{s=t^{1}+1}^{A}\left|K_{n^{(s)}, 2^{s}}\left(x^{1}, x^{2}\right)\right| d x \\
& \leq \sum_{t^{1}=0}^{m-1} \sum_{t^{2}=m+1}^{\infty} \frac{1}{2^{t^{2}}} \int_{J_{t^{1}}} \sup _{t^{2}>A \geq m} \sup _{|n|=A} \frac{1}{2^{A}} \sum_{s=t^{1}+1}^{A}\left|K_{n^{(s)}, 2^{s}}\left(x^{1}, 0\right)\right| d x^{1} \\
& \leq\left. c \sum_{t^{1}=0}^{m-1} \sum_{t^{2}=m+1}^{\infty} \frac{1}{2^{t^{2}}} \sum_{A=m}^{t^{2}} \frac{1}{2^{A}} \sum_{s=t^{1}}^{A} \int_{t^{1}}\right|_{k=n^{(s)}} ^{n^{(s)}+2^{s}-1} v_{k^{\left(t^{1}+1\right)}}\left(x^{1}\right) \widetilde{k}_{t^{1}} k d x^{1} \mid \\
& \leq c \sum_{t^{1}=0}^{m-1} \sum_{t^{2}=m+1}^{\infty} \frac{1}{2^{t^{2}}} \sum_{A=m}^{t^{2}} \frac{1}{2^{A}} \sum_{s=t^{1}}^{A}\left(\frac{1}{2^{t^{1}}}\right)^{1 / 2}\left(\frac{1}{2^{t^{1}}} 2^{s+t^{1}+2 t^{1}+2 A}\right)^{1 / 2} \\
& \leq c \sum_{t^{1}=0}^{m-1} \sum_{t^{2}=m+1}^{\infty} \frac{1}{2^{t^{2}}} \sum_{A=m}^{t^{2}} \sqrt{2^{A+t^{1}}} \leq c \sum_{t^{1}=0}^{m-1} \sum_{t^{2}=m+1}^{\infty} \sqrt{2^{t^{1}-t^{2}}} \leq c . \quad \text {. }
\end{aligned}
$$

Denote by $\overline{I_{m}^{2}}$ the complement of $I_{m}^{2}$.

LEMMA 3.

$$
\int_{\frac{I_{m}^{2}}{\mid n}} \sup _{|n| \geq m}\left|K_{n}\right| \leq c .
$$

Proof. Using trivial estimations we get

$$
\left|K_{n^{(s)}, 2^{s}}\right| \leq \sum_{k=n^{(s)}}^{n^{(s)}+2^{s}-1}\left|D_{k}\right| \leq \sum_{k=n^{(s)}}^{n^{(s)}+2^{s}-1}\left|\widetilde{k}_{t^{1}} \widetilde{k}_{t^{2}}\right| \leq 2^{s+t^{1}+t^{2} \wedge|n|},
$$

so

$$
\sum_{s=0}^{t^{1}}\left|K_{n^{(s)}, 2^{s}}\right| \leq c 2^{2 t^{1}+t^{2} \wedge|n|}
$$


From this inequality we obtain

$$
\begin{aligned}
\sum_{t^{1}=0}^{m-1} \sum_{t^{2}=t^{1}}^{m-1} \int_{t^{1} \times J_{t^{2}}} \sup _{A \geq m} \sup _{|n|=A} \frac{1}{2^{A}} \sum_{s=0}^{t^{1}}\left|K_{n^{(s)}, 2^{s}}\left(x^{1}, x^{2}\right)\right| d x \\
\leq c \sum_{t^{1}=0}^{m-1} \sum_{t^{2}=t^{1}}^{m-1} \int_{t_{t^{1}} \times J_{t^{2}}} \sup _{A \geq m} \frac{2^{2 t^{1}+t^{2} \wedge A}}{2^{A}} d x \\
\leq c \sum_{t^{1}=0}^{m-1} \sum_{t^{2}=t^{1}}^{m-1} \frac{1}{2^{t^{1}+t^{2}}} \frac{2^{2 t^{1}+t^{2}}}{2^{m}}+c \sum_{t^{1}=0}^{m-1} \sum_{t^{2}=m}^{\infty} \frac{1}{2^{t^{1}+t^{2}}} 2^{2 t^{1}} \\
\leq c \sum_{t^{1}=0}^{m-1}\left(m-t^{1}\right) 2^{t^{1}-m}+c \sum_{t^{1}=0}^{m-1} \sum_{t^{2}=m}^{\infty} 2^{t^{1}-t^{2}} \leq c
\end{aligned}
$$

and from this

$$
\sum_{t^{1}=0}^{m-1} \sum_{t^{2}=t^{1}}^{m-1} \int_{J_{t^{1}} \times J_{t^{2}}} \sup _{|n| \geq m}\left|K_{n}\right| \leq c .
$$

Similarly,

$$
\sum_{t^{2}=0}^{m-1} \sum_{t^{1}=t^{2}}^{m-1} \int_{J_{t^{1}} \times J_{t^{2}}} \sup _{|n| \geq m}\left|K_{n}\right| \leq c .
$$

Because, a.e.,

$$
\overline{I_{m}^{2}} \subseteq\left(\bigcup_{t^{2}=0}^{m-1} \bigcup_{t^{1}=t^{2}}^{m-1} J_{t^{1}} \times J_{t^{2}}\right) \cup\left(\bigcup_{t^{1}=0}^{m-1} \bigcup_{t^{2}=t^{1}}^{m-1} J_{t^{1}} \times J_{t^{2}}\right)
$$

the inequalities above imply

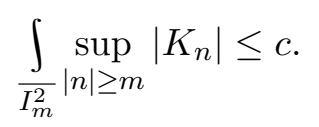

This lemma leads to the following corollary:

Corollary 4.

$$
\left\|K_{n}\right\|_{1} \leq c \quad \text { for all } n \in \mathbb{N} .
$$

The next step is to prove the quasi-local property of the maximal operator $\sigma^{*} f:=\sup _{n}\left|\sigma_{n} f\right|$.

LemMa 5. Let $f \in L^{1}\left(I^{2}\right)$ with $\int f=0$ and $\operatorname{supp} f \subseteq I_{m}\left(u^{1}\right) \times I_{m}\left(u^{2}\right)$ for some $u=\left(u^{1}, u^{2}\right) \in I^{2}$. Then

$$
\frac{\int}{I_{m}\left(u^{1}\right) \times I_{m}\left(u^{2}\right)} \sigma^{*} f \leq c\|f\|_{1} .
$$


Proof. From the shift invariance of the Haar measure we can suppose that $u^{1}=u^{2}=0$.

In the case of $|n|<m$ the situation is simple, because

$$
\sigma_{n} f(y)=\int_{I^{2}} f(\cdot) K_{n}(y-\cdot)=K_{n}(y) \int_{I_{m}^{2}} f=0 .
$$

So we can suppose that $|n| \geq m$. In this case

$$
\begin{aligned}
\frac{\int_{\frac{I_{m}^{2}}{2}} \sigma^{*} f}{} & =\int_{\frac{I_{m}^{2}}{|n| \geq m}} \sup _{I_{m}^{2}}\left|\int_{I_{m}^{2}} f(x) K_{n}(\cdot, x) d x\right| \\
& \leq \int_{I_{m}^{2}}|f(x)| \int_{\frac{I_{m}^{2}}{|n| \geq m}} \sup _{|n| \leq c}\left|K_{n}\right| \leq f \|_{1} .
\end{aligned}
$$

Proof of Theorem 1. From Corollary 4 we deduce that the maximal operator $\sigma^{*}$ is of type $(\infty, \infty)$. Lemma 5 shows that this sublinear operator is quasi-local. Using the standard method (see e.g. [7]) it follows that it is of weak type $(1,1)$. We get the statement of the theorem from the density of the 2-adic polynomials in $L^{1}\left(I^{2}\right)$. The proof of Theorem 1 is complete.

Acknowledgements. The authors would like to thank the copy editor for drawing their attention to some papers relating to convergence of the Marcinkiewicz means.

This research was supported by the Hungarian National Foundation for Scientific Research (OTKA), grant no. T 048780.

\section{References}

[1] G. Gát, On the almost everywhere convergence of Fejér means of functions on the group of 2-adic integers, J. Approx. Theory 90 (1997), 88-96.

[2] G. Grünwald, Zur Summabilitätstheorie der Fourierschen Doppelreihe, Proc. Cambridge Philos. Soc. 35 (1939), 343-350.

[3] J. G. Herriot, Norlund summability of double Fourier series, Trans. Amer. Math. Soc. 52 (1942), 72-94.

[4] E. Hewitt and K. Ross, Abstract Harmonic Analysis, Vol. I, II, Springer, Heidelberg, 1963.

[5] F. Schipp and W. R. Wade, Norm convergence and summability of Fourier series with respect to certain product systems, in: Approximation Theory (Memphis, TN, 1991), Lecture Notes in Pure Appl. Math. 138, Dekker, New York, 1992, 437-452.

[6] - - - Transforms on Normed Fields, Janus Pannonius Tudományegyetem, Pécs, 1995.

[7] F. Schipp, W. R. Wade, P. Simon, and J. Pál, Walsh Series: an Introduction to Dyadic Harmonic Analysis, Adam Hilger, Bristol and New York, 1990. 
[8] L. V. Zhizhiashvili, Generalization of a theorem of Marcinkiewicz, Izv. Akad. Nauk SSSR Ser. Mat. 32 (1968), 1112-1122 (in Russian).

Institute of Mathematics and Computer Science

College of Nyíregyháza

P.O. Box 166

H-4400 Nyíregyháza, Hungary

E-mail: blahota@nyf.hu

gatgy@nyf.hu 